\title{
Reactivation of chronic haematogenous osteomyelitis in HIV-infected patients
}

\author{
Siyo Z1 10 , Marais LC ${ }^{2}$ (두
}

1 MBChB, FC Orth SA; Department of Orthopaedics, School of Clinical Medicine, University of KwaZulu-Natal, Pietermaritzburg, South Africa

2 MBChB, FC Orth SA, MMed Ortho, PhD; Department of Orthopaedics, School of Clinical Medicine, University of KwaZulu-Natal, Pietermaritzburg, South Africa

Corresponding author: Dr Z Siyo, Department of Orthopaedic Surgery, School of Clinical Medicine, University of KwaZulu-Natal, Grey's Hospital, Pietermaritzburg, 3200, South Africa; email: zukosiyo@gmail.com

\begin{abstract}
Background: The aim of the study is to determine the prevalence of HIV infection among adult patients with reactivation of haematogenous chronic osteomyelitis.

Methods: A retrospective analysis of prospectively collected data from 143 adult patients with chronic osteomyelitis.

Results: A total of 143 patients were included in the study group, with a mean age of 38 years (range 14-78 years). Twenty-two per cent $(n=31)$ of patients were diagnosed with reactivation of chronic haematogenous osteomyelitis, while $78 \%$ of patients had contiguous chronic osteomyelitis $(29 \%$ [n=42] post-operative and $49 \%$ [ $n=70]$ post-traumatic, respectively). Forty $(28 \%)$ patients were found to be HIV positive with a mean CD4 count of $414 \mathrm{cells} / \mathrm{mm}^{3}$ (range 13-1 $034 \mathrm{cells} / \mathrm{mm}^{3}$ ). Twenty-four (60\%) of patients with HIV were on antiretroviral therapy at time of diagnosis. The prevalence of HIV infection among patients with contiguous (post-operative or post-traumatic) infections was $32 \%$, in comparison to $13 \%$ in the group with reactivation of chronic haematogenous infections ( $p=0.04$; OR 3.2; 95\% Cl 1.0-9.8).
\end{abstract}

Conclusion: The prevalence of HIV infection among patients with reactivation of chronic haematogenous osteomyelitis appeared to be lower than that seen in patients with chronic osteomyelitis from other causes and lower than that seen in the general population in South Africa.

Level of evidence: Level 4

Key words: haematogenous, osteomyelitis, HIV, AIDS

Citation: Siyo Z, Marais LC. Reactivation of chronic haematogenous osteomyelitis in HIV-infected patients. SA Orthop J 2019;18(1):21-25. http://dx.doi.org/10.17159/2309-8309/2019/v18n1a2

Editor: Prof TLB le Roux, University of Pretoria, Pretoria, South Africa

Received: December 2017

Accepted: April 2018

Published: March 2019

Copyright: () 2019 Siyo Z. This is an open-access article distributed under the terms of the Creative Commons Attribution Licence, which permits unrestricted use, distribution and reproduction in any medium, provided the original author and source are credited.

Funding: No funding was received for the purposes of performing this study.

Conflict of interest: The authors have no conflict of interest to declare. 


\section{Introduction}

The total number of people living with human immunodeficiency virus (HIV) in South Africa is currently estimated at approximately 7.06 million. In the age group 15-49 years, the national prevalence is estimated at $16.8 \% .^{1}$ The prevalence in KwaZulu-Natal, the second largest province in South Africa with a population of approximately 11.1 million people, is currently estimated at $21.5 \% .^{2} \mathrm{HIV}$ infection results in a combination of immune suppression and chronic inflammation through the mechanisms of immune exhaustion with effector T-cell dysfunction and immune senescence with premature aging of the immune system. ${ }^{3}$ The resulting neutrophil, monocyte and B-lymphocyte abnormalities lead to a decreased capacity for bacterial phagocytosis and an increased rate of bacterial infections. Methicillin-resistant Staphylococcus aureus infection is, for example, 6-18 times more common in HIV patients than in the general population. ${ }^{4} \mathrm{HIV}$ co-infection is presumed to be among the major contributing factors to the pathogenesis of bone infection.

Chronic osteomyelitis can be defined as a biofilm-based infection where the majority of pathogens are sessile-based and are resiliently attached to the nidus of infection. ${ }^{5}$ In the case of chronic haematogenous osteomyelitis, the nidus of infection is typically a sequestrum that is formed following acute osteomyelitis in childhood. The appropriate treatment of acute haematogenous osteomyelitis has resulted in a drastic decrease in the incidence of chronic osteomyelitis of haematogenous origin in the developed world; however, it remains fairly common in the developing world. ${ }^{6}$ Owing to the unique characteristics of the causative organisms, reactivation of chronic osteomyelitis may occur as much as 65 years following the initial infection. ${ }^{7,8}$ These characteristics include the internalisation of bacteria by osteoblasts which is mediated by the sigma B regulon in the case of Staphylococcus aureus. ${ }^{9}$ The exact cause of the reactivation of infection has, however, not been clearly defined but it is believed to be associated with a decrease in local or systemic immune protection. Jellis reported a possible increase in haematogenous osteomyelitis in patients with HIV infection. This was however only a comment and further data on the topic was not provided. ${ }^{10}$ To the best of our knowledge, there is currently no data on the reactivation of chronic haematogenous osteomyelitis in HIV patients.

The aim of the study is to determine the prevalence of HIV infection among adult patients with reactivation of haematogenous chronic osteomyelitis.

\section{Materials and methods}

A retrospective descriptive study was performed on prospectively collected data from consecutive patients seen at a tertiary-level tumour and sepsis unit with chronic osteomyelitis. All adult patients over the age of 14 years assessed from January 2011 to December 2014 were included in the study. Patients excluded from the study were those with atypical infections including fungal, parasitic and tuberculosis, acute post-operative infection, periprosthetic joint infection or hand sepsis.

Following ethical approval from the relevant biomedical ethics review board, data were collected with respect to patient age, cause of osteomyelitis (haematogenous or contiguous), physiological host stage and anatomic nature of the disease according to the Cierny and Mader classification system, HIV status, CD4 count and the presence of antiretroviral therapy.

For the purposes of this study chronic osteomyelitis was defined as an infection involving bone, with a duration of at least ten days, where the causative organisms were thought to have persisted either intracellularly or in interactive biofilm-based colonies. Haematogenous chronic osteomyelitis was defined as the reactivation of chronic osteomyelitis resulting from a previous episode of acute osteomyelitis of haematogenous origin. Contiguous chronic osteomyelitis was defined as chronic osteomyelitis resulting from a prior open fracture (post-traumatic) or operative intervention (post-operative).

All patients were screened for HIV infection. Following clinical, radiological and biochemical evaluation, patients were classified according to a modified version of the original Cierny and Mader classification system (Table I). ${ }^{11}$ In terms of the physiological status of the host, the Cierny and Mader classification system was modified in order to provide a more pragmatic and objective definition of a $\mathrm{C}$ host. A patient was classified as a $\mathrm{C}$ host if one major or more than two minor risk factors were present (Table II). In order to remove any ambiguity during classification of the anatomical nature of the disease, this was performed prior to, rather than following, the debridement.

Statistical analysis was performed using Stata 13.0 (StataCorp. College Station, Texas). Continuous variables were reported as mean $( \pm S D$ ) or median (with interquartile range) and categorical variables as numbers and percentages, unless otherwise stated. Categorical data were compared using the Fisher's exact test or the chi-square test. All tests were two-sided, and the level of significance was set at $p<0.05$.

Table I: Modified version of the original Cierny and Mader classification system that served to guide treatment strategy selection ${ }^{11}$

\begin{tabular}{|l|l|}
\multicolumn{1}{|c|}{ Classification } & \multicolumn{1}{c}{ Characteristic } \\
\hline Physiological & No risk factors \\
\hline Type A host & Fewer than three minor risk factors \\
\hline Type B host & One major and/or three or more minor risk factors \\
\hline Pathoanatomy & \\
\hline I - Medullary & No cortical sequestration \\
\hline II - Cortical & Direct contiguous involvement of cortex only \\
\hline III - Combined (stable) & Both cortex and medullary regions involved \\
\hline IV - Combined (unstable) & As for III plus unstable prior to debridement \\
\hline Nidus & \\
\hline Sequestrum & Cortical sequestrum present \\
\hline Implant & Biofilm-based infection in presence of implant \\
\hline No identifiable nidus & Minimal necrosis osteomyelitis \\
\hline Impairment & \\
\hline Minimal & Patient able to perform ADL (activities of daily living) \\
\hline Severe & Unable to perform ADL \\
\hline
\end{tabular}


Table II: Risk factors used to stratify the physiological status of the host ${ }^{11}$

\section{Major risk factors}

CD4 count $<350$ cells $/ \mathrm{mm}^{3}$

Albumin $<30 \mathrm{~g} / \mathrm{L}$

$\mathrm{HbA} 1 \mathrm{C} \geq 8 \%$

Cellulitis or abscess

Malignancy at site of infection

Pathological fracture
Minor systemic risk factors

HIV infection

Anaemia

Smoking

Diabetes mellitus

Rheumatoid arthritis

Chronic lung disease

Chronic cardiac failure

Paraplegia/quadriplegia

Drug or substance abuse

Chronic corticosteroid use

Active tuberculosis

Ischaemic heart disease

Cerebrovascular disease

Compliance and motivation

Age $>65$ years

Common variable immune deficiency
Minor local risk factors

Poor soft tissues requiring flap

Chronic venous insufficiency

Peripheral vascular disease

Previous radiation therapy

Surgery will result in instability

Adjacent joint stiff/arthritic

Heterotopic ossification

Failed reconstruction elsewhere

Foot involvement

Pelvic involvement

Adjacent joint involved

Segmental resection of $\geq 6 \mathrm{~cm}$ required to achieve cure

\section{Results}

A total of 149 patients met the inclusion criteria. Four patients with early post-operative infection and two patients with fungal osteomyelitis were excluded, leaving a total of 143 patients in the study group. The mean age of patients was 38 years (range 14-78; standard deviation [SD] 15.5 years). Twenty-eight per cent $(n=40)$ of patients were found to be HIV positive with a mean CD4 count of 414 cells $/ \mathrm{mm}^{3}$ (range 13-1 034; SD 132 cells $/ \mathrm{mm}^{3}$ ). Sixty per cent $(n=24)$ of patients with HIV were on antiretroviral therapy at time of diagnosis.

Twenty-two per cent $(n=31)$ of patients were diagnosed with reactivation of chronic haematogenous osteomyelitis, while $78 \%$ of patients had contiguous chronic osteomyelitis (29\% [ $n=42]$ post-operative and $49 \%$ [ $n=70]$ post-traumatic, respectively). The location of the infection was the tibia in $52 \%$ of cases $(n=75)$, femur in $27 \%(n=39)$, humerus, pelvis or foot in $5 \%(n=7)$, fibula or radius/ ulna in $3 \%(n=5)$ and clavicle in $1 \%$ of cases. Overall, $15 \%(n=21)$ of patients were classified as A hosts, $41 \%(n=59)$ were $B$ hosts and $44 \%(n=63) C$ hosts. Of the B hosts, nine patients were HIV-positive with a mean CD4 of 627 cells $/ \mathrm{mm}^{3}$. Thirty-one (49\%) of C hosts were HIV-positive, mean CD4 352 cells $/ \mathrm{mm}^{3}$.

The prevalence of HIV infection among patients with contiguous (post-operative or post-traumatic) infections was $32 \%$, in comparison to $13 \%$ in the group with reactivation of chronic haematogenous infections ( $p=0.04$; OR 3.2; 95\% Cl 1.0-9.8) (Table III). In addition, there was a significant difference between the two groups in terms of the site of infection, the physiological stage of the host and the anatomic nature of the disease (Table III). Two of the four HIV patients in the haematogenous group (mean CD4 $487 \mathrm{cells} / \mathrm{mm}^{3}$ ) were on antiretroviral medication compared to 12 of the 28 patients in the contiguous group (mean CD4 405 cells $/ \mathrm{mm}^{3}$ ).

\section{Discussion}

Considerable controversy remains regarding the association of HIV infection and the development of bone infections. In the 1990s, Jellis and Hoekman independently reported an infection rate of operatively treated fractures of $24 \%$ and $33 \%$ in symptomatic HIV patients. ${ }^{10,12}$ In contrast, a study by Harrison et al., in 2002, showed that the risk of post-operative infection is dependent on wound contamination. HIV status was not found to be a risk factor for wound infection following operative management of closed fractures. ${ }^{13}$ The study reinforced earlier findings that asymptomatic HIV-positive patients with high energy open injuries were prone to infection compared to HIV negative. ${ }^{14}$ In contrast to this, Howard et al. showed that HIV does not necessarily increase early infection in open fractures. ${ }^{15}$ These findings were echoed by Niewoudt et al., who noted that HIV did not appear to be associated with an increased risk of deep infection or non-union in grade III open tibia fractures treated with circular external fixation. ${ }^{16}$ The influence of CD4 count on the development of infection also remains unclear. Guild et al., showed an increased infection rate in patients with a CD4 count below $300 .{ }^{17}$ All of the above-mentioned studies, however, focused on contiguous (post-operative or post-traumatic) infections. Limited data is available on the impact of HIV on haematogenous osteomyelitis.

Lavy and co-workers noted a three-fold increase in the number of septic cases treated in Malawi and speculated that this may, at least in part, have been the result of an increased seroprevalence of HIV. ${ }^{18}$ While osteomyelitis was mentioned in this report, haematogenous osteomyelitis was not specifically looked at. In 1996 Jellis reported an increase in the incidence of adult long-bone haematogenous osteomyelitis in patients with HIV and further stated that it was a common orthopaedic presentation of adults with advanced HIV disease. ${ }^{10}$

The aim of this study was to determine the prevalence of HIV infection among adult patients presenting with chronic haematogenous osteomyelitis in an attempt to investigate the possible association between HIV infection and adult chronic osteomyelitis. Intuitively, it seems reasonable to expect that an immune-compromising disease like HIVIAIDS might cause an increase in the incidence of reactivation of quiescent adult osteomyelitis, especially in patients with very low CD4 counts.

Somewhat surprisingly we found a lower prevalence of HIV infection among adult patients presenting with chronic haematogenous osteomyelitis in comparison to adult osteomyelitis from other causes $(13 \%$ vs $32 \%, p=0.04)$. The prevalence of HIV infection in the contiguous group of patients was comparable to that seen in the general population of the region where the study was performed; however, in the haematogenous group it was considerably lower. In addition, the HIV-positive patient who did present with haematogenous osteomyelitis did not have 
Table III: Comparative statistics of patients with reactivation of chronic haematogenous osteomyelitis and contiguous osteomyelitis

\begin{tabular}{|c|c|c|c|}
\hline & $\begin{array}{c}\text { Haematogenous } \\
\text { chronic osteomyelitis } \\
(n=31)\end{array}$ & $\begin{array}{c}\text { Contiguous } \\
\text { chronic osteomyelitis } \\
(n=112)\end{array}$ & p-value \\
\hline Site & & & $0.01^{\mathrm{iii}}$ \\
\hline Tibia & $12(39 \%)^{i}$ & $63(56 \%)$ & \\
\hline Femur & $15(48 \%)$ & $24(21 \%)$ & \\
\hline Humerus & - & $7(6 \%)$ & \\
\hline Other & $4(13 \%)$ & $18(16 \%)$ & \\
\hline Host staging ${ }^{12}$ & & & $<0.01^{i i i}$ \\
\hline A-host & $13(42 \%)$ & $8(7 \%)$ & \\
\hline B-host & $10(32 \%)$ & $49(44 \%)$ & \\
\hline C-host & $8(26 \%)$ & 55 (49\%) & \\
\hline Anatomic classification ${ }^{12}$ & & & $<0.01^{\mathrm{iii}}$ \\
\hline Type 1 & - & $3(3 \%)$ & \\
\hline Type 2 & - & $2(2 \%)$ & \\
\hline Type 3 & $26(84 \%)$ & $44(39 \%)$ & \\
\hline Type 4 & $5(16 \%)$ & $63(56 \%)$ & \\
\hline HIV status & & & $0.04^{\text {iv }}$ \\
\hline Positive & $4(13 \%)$ & $36(32 \%)$ & \\
\hline Negative & $27(87 \%)$ & $76(68 \%)$ & \\
\hline CD4 count $^{\text {vi }}$ & $487(360-646)^{i i}$ & $405(13-1034)$ & $0.47^{v}$ \\
\hline
\end{tabular}

(i) $n$ (\%); (ii) mean (range); (iii) chi-square test; (iv) Fisher's exact test (v) t-test; (vi) CD4 count of HIV-positive patients in each group

exceptionally low CD4 counts. While this study by no means provides the definitive answer, it appears that HIV infection may not necessarily be associated with the reactivation of quiescent haematogenous osteomyelitis in adults, as was initially thought.

This study has several shortcomings. Due to the retrospective nature of the study it was not possible to determine how many patients with haematogenous osteomyelitis remained asymptomatic. Thus, we were unable to compare the true prevalence of reactivation in HIV-positive and -negative patients. A long-term prospective follow-up of patients with haematogenous osteomyelitis will be required for this purpose. A further limitation is the small sample size, especially in the haematogenous group. The question therefore remains unanswered and further research in the field is warranted.

\section{Conclusion}

The prevalence of HIV infection among patients with reactivation of chronic haematogenous osteomyelitis appeared to be lower than that seen in patients with chronic osteomyelitis from other causes, and lower than that seen in the general population in South Africa. This appears to be in contradiction to previous reports stating that HIV infection may be associated with adult chronic haematogenous osteomyelitis.

\section{Ethics statement}

Prior to commencement of the study ethical approval was obtained from the following ethical review boards:

1. KwaZulu-Natal Department of Health (KZ_2016RP44_836)

2. Biomedical Research Ethics Committee (BREC 204/16)

All procedures were in accordance with the ethical standards of the responsible committee on human experimentation (institutional and national) and with the Helsink Declaration of 1975, as revised in 2008.

Informed written consent was not obtained from all patients included in the study.

\section{Declarations}

The authors declare authorship of this article and that they have followed sound scientific research practice. This research is original and does not transgress plagiarism policies.

\section{Acknowledgements}

None

\section{Author contributions}

ZS: Literature review conceptualisation, design, data collection and analysis, manuscript.

LCM: Conceptualisation, design, data collection and analysis, manuscript.

\section{ORCID}

Siyo Z (D) http://orcid.org/0000-0002-8420-9244

Marais LC (D) http://orcid.org/0000-0002-1120-8419

\section{References}

1. Statistics SA. Mid-year population estimates 2017. http://www. statssa.gov.za/publications/P0302/P03022017.pdf (date last accessed 30 November 2017)

2. Welz T, Hosegood V, Jaffar S, Batzing-Feigenbaum J, Herbst K, Newell M. Continued very high prevalence of HIV infection in rural KwaZulu-Natal, South Africa: a population-based longitudinal study. AIDS 2007;21:1467-72.

3. Mohan T, Bhatnagar S, Gupta DL, Rao DN. Current understanding of HIV-1 and TCcell adaptive immunity: Progress to date. Microb Pathogen 2014;73:60-69.

4. Hidron Al, Kemper R, Moanna A, Rimland D. Methicillin-resistant Staphylococcus aureus in HIV-infected patients. Inf Drug Resist 2010;3:73-86.

5. Cierny G. Surgical treatment of osteomyelitis. Plast Reconstr Surg 2011;127(Suppl 1):S190-94

6. Walter G, Kemmerer M, Kappler C, Hoffmann R. Treatment algorithms for chronic osteomyelitis. Dtsch Arztebl Int 2012;109(14):257-64. 
7. Al-Maiyah M, Hemmady MV, Shoaib A, Morgan-Jones RL. Recurrence of chronic osteomyelitis in a regenerated fibula after 65 years. Orthopedics 2007;30:403-404.

8. Donati L, Quadri P, Reiner M. Reactivation of osteomyelitis caused by Staphylococcus aureus after 50 years. J Am Geriatr Soc 1999;47:1035-37.

9. Nair SP, Bischoff M, Senn MM, et al. The sigma B regulon influences internalization of Staphylococcus aureus by osteoblasts. Infect Immun 2003;71:4167-70.

10. Jellis JE. Orthopaedic surgery and HIV disease in Africa. Int Orthop 1996;20:253-56

11. Marais LC, Ferriera N, Aldous C, le Roux TLB. The outcome of treatment of chronic osteomyelitis following an integrated approach. Strat Traum Limb Recon 2016;11(2):135-42.

12. Hoekman P, Van Den Perre P, Nellisen J, Kwisanga B, Bogaerts $J$, Kanyangabo F. Increased frequency of infection after open reduction of fractures in patient who are seropositive for human immunodeficiency virus. J Bone Joint Surg Am 1991;73ロA(5):675-79.

13. Harrison WJ, Lavy CBD, Lewis CP. One-year follow-up of orthopaedic implants in HIV-positive patients. Int Orthop 2004;28:329-32.

14. Paiement GD, Hymes RA, La Douceur MS, Gosselin RA, Green HD. Postoperative infections in asymptomatic HIV-seropositive orthopedic trauma patients. J Trauma 1994;37(4):545-50.

15. Howard NE, Phaff M, Aird J, Wicks L, Rollinson P. Does human immunodeficiency virus status affect early wound healing in open surgically stabilized tibial fractures? Bone Joint $J$ 2013;95-B:1703-707.

16. Niewoudt L, Ferreira N, Marais LC. Short term results of grade III open tibia fractures treated with circular fixators. S Afr Orthop J 2016;15(3):20-26.

17. Goerge N, Guild MD, Thomas J, Moore MD, Whitney Barnes MPH, Christopher BS. CD4 count is associated with postoperative infection in patients with orthopaedic trauma who are HIV positive. Clin Orthop Relat Res 2012;470:1507-12.

18. Lavy C, Schmidt C, Kalua E, Phuka J. The resistable rise of surgical sepsis in Malawi. Malawi Med J 2001;13(1):35-36. 